\title{
Clinical characteristics and risk factors associated with nosocomial COVID-19 infection in patients with hematological disorders in Japan
}

\author{
Shin Ohara ${ }^{1}\left[\right.$ - Takayuki Fujii ${ }^{1} \cdot$ Shiro Ide $^{1} \cdot$ Tomoyuki Uchida $^{1} \cdot$ Morihiro Inoue $^{1} \cdot$ Masao Hagaihara $^{1}$
}

Received: 23 November 2020 / Revised: 6 June 2021 / Accepted: 9 June 2021 / Published online: 21 June 2021

(c) Japanese Society of Hematology 2021

\begin{abstract}
Patients with cancer are considered at high risk of acquiring coronavirus disease (COVID-19). To identify patients who are likely to be diagnosed with severe COVID-19, we analyzed the risk factors for mortality in patients admitted to the hematology department at our institute. The mortality rate of all patients was as high as $62 \%$ ( 21 of the 34 patients), and most of these patients had malignant malignancies. Patients before an achievement of remission had a 10.8-fold higher risk of death than those in remission. The group receiving chemotherapy with steroids had a shorter survival time and had an 8.3-fold higher risk of death than that receiving chemotherapy without steroids. During the COVID-19 pandemic, it is necessary to carefully monitor or follow-up patients with active diseases and patients receiving steroid-containing chemotherapy.
\end{abstract}

Keywords COVID-19 · Hematological disorders · Chemotherapy $\cdot$ Nosocomial infection

\section{Introduction}

Severe acute respiratory syndrome coronavirus 2 (SARS$\mathrm{CoV}-2$ ) and its resulting illness, coronavirus disease (COVID-19), spread rapidly worldwide. Moreover, in Japan, the cumulative number of individuals diagnosed with COVID-19 exceeded 80,000, and more than 1500 individuals have died at the end of September. In our institute, a large number $(n=109)$ of hospitalized patients were diagnosed with nosocomial COVID-19 infection, which is considered the largest cluster except for an outbreak on a cruise ship [1].

During these past few months, several risk factors predisposing to severe disease status or death due to COVID19 have been identified [2-9]. Among them, patients with active cancer have been reported to have an increased risk of acquiring COVID-19, with more severe conditions resulting in intensive care unit admission or even death [5]. Furthermore, patients with hematological malignancies, such as leukemia, lymphoma, and multiple myeloma, have immunodeficiency because of the disorder itself and anticancer or immunosuppressive treatments. Furthermore, several studies indicated that individuals with hematological cancer have a

Shin Ohara

shin.md.o@gmail.com

1 Eiju General Hospital, 2-23-16, Higashi-Ueno, Taito, Tokyo, Japan significantly higher mortality rate than individuals without non-hematological cancer [2]. Nevertheless, data regarding patients diagnosed with nosocomial infection with hematological diseases are insufficient. Here, we characterized the outcomes of our patients diagnosed with COVID-19 and analyzed the risk factors for mortality. We previously reported "Retrospective analysis of nosocomial COVID-19: a comparison between patients with Hematological disorders and other diseases" in the past, an overview is given below. Among the patients with hematological disease and those with other diseases, mortality were 52.5 and $35.1 \%$, respectively, median overall survival of the patients with hematological disease was poor (29 days; $P=0.078$ ) [10]. In this report, we excludes those who believe they have died of the underlying illness or who have been transferred to another hospital and are unable to follow up in detail.

\section{Materials and methods}

Forty-eight out of the 61 adult patients with hematological diseases were admitted to the Hematology Department of the Eiju General Hospital. From 21 March to 15 April 2020, patients who had positive SARS-CoV-2 RNA results by reverse transcriptase quantitative polymerase chain reaction obtained from the patient's nose or throat swabs were enrolled. After excluding cases, which were asymptomatic, 
transferred to other hospitals for COVID-19 treatment or have died due to the underlying hematological illness, 34 cases were selected for the analysis. Data were retrospectively collected from patients' electronic medical records. Treatments for COVID-19 included the administration of favipiravir, nafamostat, and ciclesonide based on the attending physician's discretion. Outcomes were categorized as either survival or non-survival at the final observation period.

The Kaplan-Meier analysis (log-rank test) and Cox proportional hazards models were used to analyze survival data. Risk factors associated with death and their odds ratios (ORs) were analyzed using the Fisher's exact test. Factors with $P$ values $<0.05$ from univariate analysis were examined for multivariate analysis. $P$ value $<0.05$ was considered statistically significant. All statistical analyses were performed using EasyR.

\section{Results}

The characteristics of recovered or dead patients and the OR of death are summarized in Table 1 . Of the 34 patients, 11 , $7,7,4,1,1,2$, and 1 patients were diagnosed with malignant lymphoma, multiple myeloma, myelodysplastic syndrome, acute myeloid leukemia, acute lymphoid leukemia, myeloproliferative disorders, immune thrombocytopenia (ITP), and pure red cell aplasia, respectively. A total of 19 (56\%) were male, and the patient's median age was 72.5 years (60-90 years). The percentage of elderly people was relatively high, with $79 \%$ aged over 70 years. Among the 31 (91\%) patients with underlying hematological malignancies, 22 and 9 patients were before an achievement of remission and in remission, respectively. Twenty-five patients had recently received chemotherapy, of which 15 with steroidcontaining regimens.

The median follow-up period was 21.5 days, and 21 out of the $34(62 \%)$ patients died due to COVID-19 infection, mainly due to respiratory distress, while 13 (38\%) patients recovered (Fig. 1a). The median overall survival was 21.5 days. Patients before an achievement of remission had a significantly higher risk of death and significantly shorter survival time ( 15.5 days vs. NR, $P=0.00494$ ) than those in remission (OR 10.8, $P=0.0118$, Fig. $1 b$ ). The group receiving chemotherapy with steroids had significantly shorter survival time and higher risk of death $(\mathrm{OR} 8.35, \mathrm{p}=0.0344)$ than that receiving chemotherapy without steroids (19 days vs. NR, $P=0.0258$, Fig. 1c). The group with lymphoid had shorter survival time than the group with myeloid malignancies without a statistical significance (20 days vs. NR, $P=0.172$, Fig. 1d). The group with hypertension as comorbidity tended to show shorter survival time than that with no hypertension (13 vs. 21 days, $P=0.0812$, Fig. 1e). There was no difference in survival time with or without other associated disorders. No statistically significant risk has been detected as for gender, age, number of white blood cells or lymphocytes, values of lactic acid dehydrogenase or IgG at the onset of disorder.

\section{Discussion}

Several studies have reported the clinical effects of COVID19 in patients with cancer. Kuderer et al. [2] have reported the outcomes of 928 patients with cancer in the United States. In their analysis, the mortality rate of patients with hematological malignancies was equivalent to that of patients with solid tumors. In contrast, Yang et al. from China reported that patients diagnosed with hematological malignancies had a significantly poorer prognosis than those with solid tumors (41 vs. 17\%) [11]. A recent report from the United Kingdom also demonstrated that patients with hematological malignancies had a more severe COVID-19 clinical trajectory, including a requirement for intensive supportive interventions or even death, compared to those with solid organ tumors [9]. Several other published reports are summarized in Table 2 [2-9, 12-15], which demonstrated an average mortality rate of $40 \%$. In our hospital, although the mortality rate was $43 \%$ ( 21 out of the 48 infected patients), the rate increased to $62 \%$ if patients with asymptomatic, transferred to the other institutions or those have died due to the underlying hematological illness, were excluded from the analysis. Nosocomial outbreaks in our hospital have spread viruses to relatively frail and elderly patients requiring hospitalization during the short period and also many cases with intractable basic hematological illness were included; all of which contributed to huge numbers of critical cases.

Of the 34 patients, 31 had hematological malignancies, and 2 of the remaining 3 patient received high doses of steroids for ITP and died due to the aggressive development of acute respiratory distress. Characteristics associated with COVID-19-infected rheumatic patients have been reported by Gianfrancesco et al., who reported that a prednisolone dose $\geq 10 \mathrm{mg}$ /day was associated with higher odds of hospitalization [16]. Although a history of steroid therapy in the past month did not affect patients' survival, caution should be paid to patients receiving immunosuppressed therapies not only with steroids but also with recent several immunomodulating agents such as monoclonal antibodies or molecular target drugs.

Of the remaining 31 patients with malignancies, 19 $(61 \%)$ have died, which was still considered a higher rate of mortality. Our study has several important findings. First, the risk of death was significantly higher and the survival time was shorter in cases not yet achieved a remission than those in a remission regarding background hematological 
Table 1 Demographics and baseline characteristics of patients with coronavirus disease 2019 and odds ratio of death

\begin{tabular}{|c|c|c|c|c|}
\hline & Non-survival & Survival & Odds ratio & $P$ value \\
\hline Total & 21 & 13 & & \\
\hline \multicolumn{5}{|l|}{ Gender } \\
\hline Male & 10 & 9 & $0.415(0.070-2.102)$ & 0.296 \\
\hline Female & 11 & 4 & & \\
\hline \multicolumn{5}{|l|}{ Age } \\
\hline$\geq 70$ & 16 & 11 & $0.591(0.048-4.471)$ & 0.682 \\
\hline$<70$ & 5 & 2 & & \\
\hline$\geq 80$ & 6 & 3 & $1.322(0.216-10.099)$ & 1 \\
\hline$<80$ & 15 & 10 & & \\
\hline \multicolumn{5}{|l|}{ Disease } \\
\hline Cancer & 19 & 12 & $0.797(0.012-16.907)$ & 1 \\
\hline Non cancer & 2 & 1 & & \\
\hline \multicolumn{5}{|l|}{ White blood cells $/ \mu$ l } \\
\hline$<4000$ & 8 & 6 & $0.725(0.143-3.668)$ & 0.728 \\
\hline$\geq 4000$ & 13 & 7 & & \\
\hline \multicolumn{5}{|l|}{ Lymphocytes/ $\mu 1$} \\
\hline$<1000$ & 17 & 8 & $2.576(0.425-17.009)$ & 0.254 \\
\hline$\geq 1000$ & 4 & 5 & & \\
\hline \multicolumn{5}{|l|}{ Lactate dehydrogenase/l } \\
\hline$\geq 250$ & 7 & 3 & $1.642(0.281-12.286)$ & 0.704 \\
\hline$<250$ & 14 & 10 & & \\
\hline \multicolumn{5}{|l|}{$\mathrm{IgG}, \mathrm{mg} / \mathrm{dl}$} \\
\hline$\geq 500$ & 15 & 11 & $0.283(0.00530-3.073)$ & 0.37 \\
\hline$<500$ & 5 & 1 & & \\
\hline \multicolumn{5}{|l|}{ Remission } \\
\hline No & 17 & 5 & $10.758(1.459-138.874)$ & 0.0118 \\
\hline Yes & 2 & 7 & & \\
\hline \multicolumn{5}{|l|}{ Subtypes of malignancies } \\
\hline Lymphoid & 14 & 5 & $3.734(0.667-23.879)$ & 0.13 \\
\hline Myeloid & 5 & 7 & & \\
\hline \multicolumn{5}{|l|}{ Chemotherapy } \\
\hline With prednisolone & 12 & 3 & $8.350(1.111-88.068)$ & 0.0344 \\
\hline Without prednisolone & 3 & 7 & & \\
\hline \multicolumn{5}{|l|}{ Hypertension } \\
\hline Yes & 10 & 3 & $2.933(0.535-21.415)$ & 0.2763 \\
\hline No & 11 & 10 & & \\
\hline \multicolumn{5}{|l|}{ Diabetes } \\
\hline Yes & 7 & 4 & $1.121(0.206-6.814)$ & 1 \\
\hline No & 14 & 9 & & \\
\hline
\end{tabular}

malignancies. Kuderer et al. [2] have also shown that advanced, specifically progressing, patients experiencing non-remission had higher mortality rate than patients in remission. In addition, Passamonti et al. [8] reported that older age, progressive disease status, and several disease types, such as acute myeloid leukemia, non-Hodgkin lymphoma, and plasma cell neoplasms, were significant risk factors from a large database of 536 hematological patients.

Second, the group receiving chemotherapy with steroids had a significantly higher risk of death and shorter survival time than the group receiving chemotherapy alone. As a result, lymphoid malignancies tended to show a shorter survival than myeloid ones because steroid-containing chemotherapies were preferentially administered for malignant lymphoma and myeloma. In contrast, Lee LYW has shown that patients with leukemia, but not with lymphoma, showed a significantly higher case fatality rate [9]. It is assumed that chronic lymphoid leukemia, which is the most commonly diagnosed leukemia in the Western world but rare in Asia, including our country [17], has been reported to 


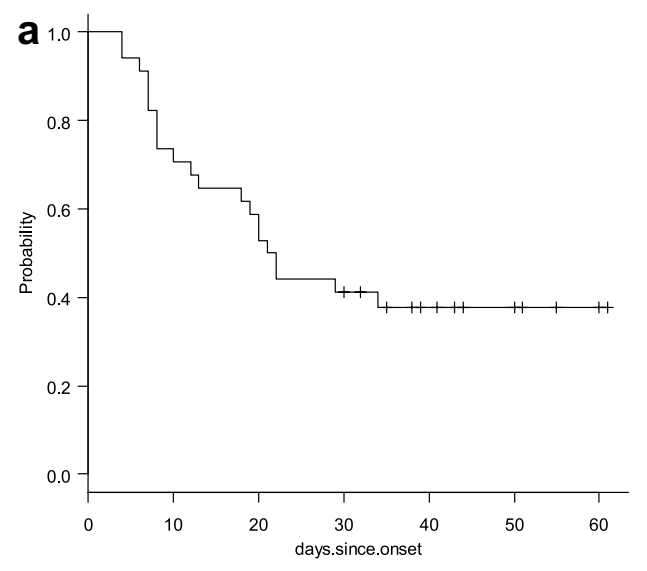

b
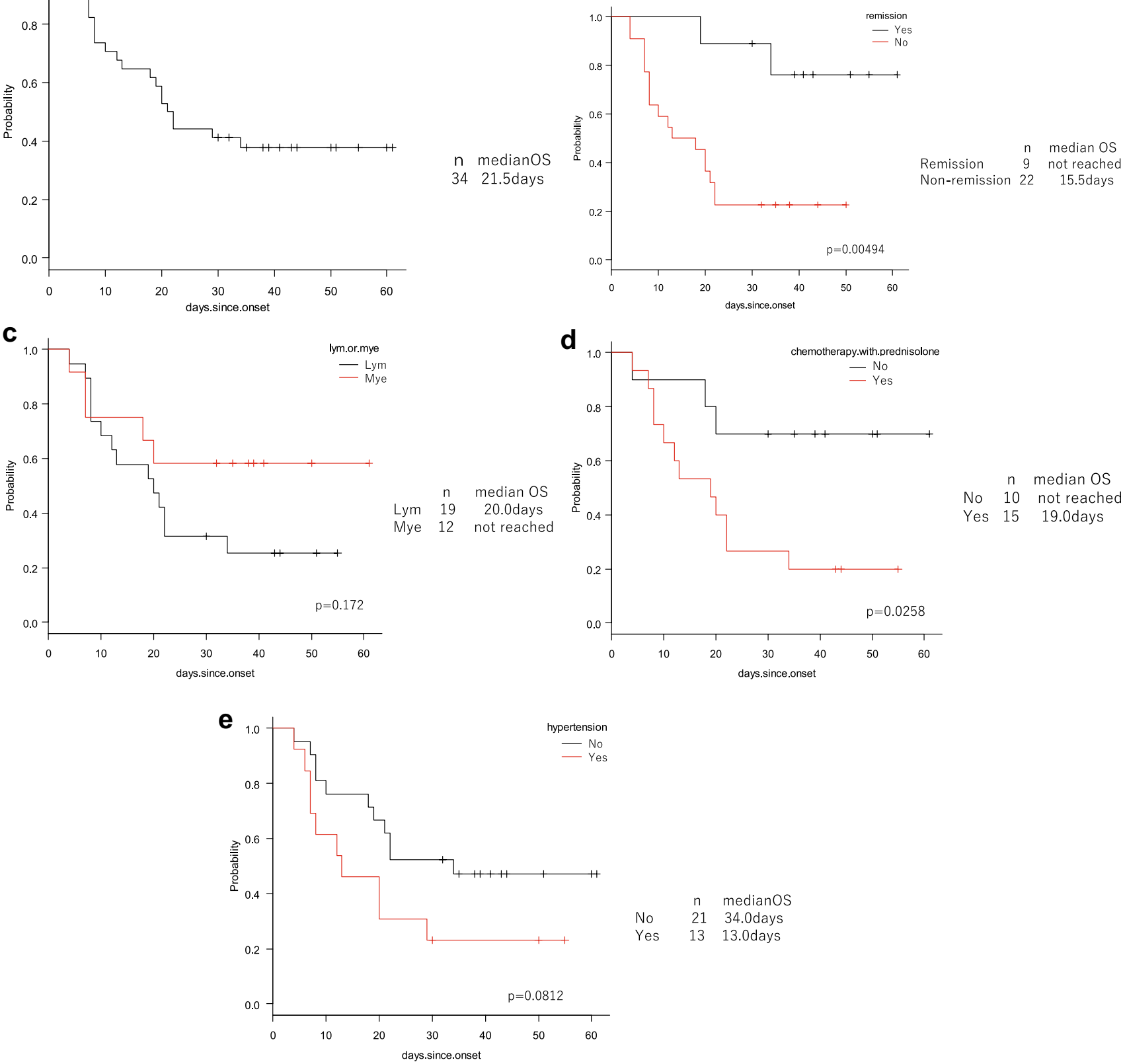

Fig. 1 The Kaplan-Meier curve for survival. a All patients, $\mathbf{b}$ remission or non-remission, $\mathbf{c}$ chemotherapy with or without prednisolone, $\mathbf{d}$ subtypes of malignancies (lymphoid or myeloid), e presence or absence of hypertension

be at particularly high risk of infection and poor outcome, leading to an increased fatality rate, which might have contributed to the inconsistency with our results. Several other groups have also shown that intensive immunosuppressive or cytotoxic treatment increased the risk of death $[5,6,9]$. However, to the best of our knowledge, the present study is the first to demonstrate the risk of steroid-containing chemotherapeutic regimens. From these results, it was revealed that the decrease in cell-mediated immunity, which was prompted by a combined medication of chemotherapeutical reagents and steroids had a significant effect on the severity of COVID-19.

It is widely acknowledged that lymphocytes count at disease onset estimate the prognosis in cases other than hematological disorder [5]. In our report, patients with low lymphocytes count at the onset tended to have shorter survival time without statistical significance. It was reasoned that already at the moment of exposure to SARS-CoV2, hematopoietic 
Table 2 Comparison of our patients with previous reports on mortality and identified mortality risks

\begin{tabular}{llllll}
\hline Author & Country & Case number & Died (\%) & Risk factors & \\
\hline Kuderer [2] & USA & 204 & 14 & Cancer status & \\
He [11] & China & 13 & 62 & \\
Dai [12] & China & 9 & 33 & CRP & \\
TA [3] & UK & 55 & 35 & Cancer status & PS \\
Martin-Moro [4] & Spain & 34 & 32 & Intensity of Txp & \\
Shah [5] & UK & 80 & 39 & Chemo Txp & \\
Booth [6] & UK & 66 & 51 & & ARDS \\
Aries [13] & UK & 35 & 40 & Age(> 70 years) & CRP(> 10) \\
Malard [14] & France & 24 & 40 & Age & Disease status \\
Sanchez-Pina [7] & Spain & 39 & 35.9 & 37 & \\
Passamonti [8] & Italy & 536 & & Recent Txp & Chemo containg steroid \\
& & & 41 & Cancer status & MM \\
Lee [9] & UK & 224 & 62 & &
\end{tabular}

malignancies themselves or immunosuppression by chemotherapy had decreased numbers of lymphocytes on the whole. The number of lymphocyte count decreased in parallel with clinical severity (data now shown), which should have caused dismal outcomes.

This study has the following limitations. (1) This study comprised several patients who were relatively young and non-severely transferred to other hospitals; therefore, the mortality rate might have been overestimated due to the large number of elderly patients. (2) The sample size was significantly small to draw a definitive conclusion. Despite these limitations, we proposed the following instructions. Patients with hematological malignancies before an achievement of remission or patients undergoing chemotherapy including steroids, have a significantly high risk of acquiring a severe course of COVID-19. These high-risk patients should be more strictly followed up for clinical signs of COVID-19 or should be intensively monitored at the time of admission. Choosing a steroid-free or reduced chemotherapy regimen, specifically for lymphoid malignancies, might be considered as another temporal option.

\section{Declarations}

Conflict of interest The authors declare that they have no conflict of interest.

\section{References}

1. Yoshimura Y, Sasaki H, Horiuchi H, Miyata N, Tachikawa N. Clinical characteristics of the coronavirus disease 2019
(COVID-19) outbreak on a cruise ship. J infect Chemother. 2020;26:1177-80.

2. Kuderer NM, Choueiri TK, Shah DP, Shyr Y, Rubinstein SM, Rivera DR, et al. Clinical impact of COVID-19 on patients with cancer (CCC19): a cohort study. Lancet. 2020;395:1907-18.

3. Fox TA, Troy-Barnes E, Kirkwood AA, Chan WY, Day JW, Chavda SJ, et al. Clinical outcomes and risk factors for severe COVID-19 infection in patients with haematological disorders receiving chemo- or immunotherapy. Br J Haematol. 2020;191:194-206.

4. Martín-Moro F, Marquet J, Piris M, Michael BM, Sáez AJ, Corona M, et al. Survival study of hospitalised patients with concurrent COVID-19 and haematological malignancies. Br J Haematol. 2020;190:e16-20.

5. Shah V, Ko TK, Zuckerman M, Vidler J, Sharif S, Mehra V, et al. Poor outcome and prolonged persistence of SARS-CoV-2 RNA in COVID-19 patients with haematological malignancies; King's College Hospital experience. Br J Haematol. 2020;190:e279-82.

6. Booth S, Willan J, Wong H, Khan D, Farnell R, Hunter A, et al. Regional outcomes of severe acute respiratory syndrome coronavirus 2 infection in hospitalised patients with haematological malignancy. Eur J Haematol. 2020;105:476-83.

7. Sanchez-Pina JM, Rodriguez MR, Quismondo NC, Manso RG, Colmenares R, Also DG, et al. Clinical course and risk factors for mortality from COVID-19 in patients with haematological malignancies. Eur J Haematol. 2020. https://doi.org/10.1111/ejh. 13493.

8. Passamonti F, Cattaneo C, Arcaini L, Bruna R, Cavo M, Merli $\mathrm{F}$, et al. Clinical characteristics and risk factors associated with COVID-19 severity in patients with haematological malignancies in Italy: a retrospective, multicentre, cohort study. Lancet Haematol. 2020;:S2352-3026(20):30251-9.

9. Lee LYW, Cazier J, Starkey T, Briggs SEW, Arnold R, Bisht $\mathrm{V}$, et al. COVID-19 prevalence and mortality in patients with cancer and the effect of primary tumour subtype and patient demographics: a prospective cohort study. Lancet Oncol. 2020;2045(20):30442-3.

10. Uchida T, Takagi Y, Mizuno A, Okamura H, Saito H, Ide S, et al. Retrospective analysis of nosocomial COVID-19: a comparison between patients with hematological disorders and other diseases. Rinsho Ketsueki. 2020;61(8):857-64. 
11. Yang K, Sheng Y, Huang C, Jin Y, Xiong N, Jiang K, et al. Clinical characteristics, outcomes, and risk factors for mortality in patients with cancer and COVID-19 in Hubei, China: a multicentre, retrospective, cohort study. Lancet Oncol. 2020;21:904-13.

12. He W, Chen L, Chen L, Yuan G, Fang Y, Chen W, et al. COVID-19 in persons with haematological cancers. Leukemia. 2020;34:1637-45.

13. Dai M, Liu D, Liu M, Zhou F, Li G, Chen Z, et al. Patients with cancer appear more vulnerable to SARS-CoV-2: a multicenter study during the COVID-19 outbreak. Cancer Discov. 2020;10:783-91.

14. Aries JA, Davies JK, Auer RL, Hallam SL, Montoto S, Smith M, et al. Clinical outcome of coronavirus disease 2019 in haematooncology patients. Br J Haematol. 2020;190:e64-7.

15. Malard F, Genthon A, Brissot E, Wyngaert Z, Marjanovic Z, Ikhlef S, et al. COVID-19 outcomes in patients with hematologic disease. Bone Marrow Transplant. 2020;6:1-5.
16. Gianfrancesco M, Hyrich KL, Al-Adely S, Carmona L, Danila MI, Gossec L, et al. Characteristics associated with hospitalisation for COVID-19 in people with rheumatic disease: data from the COVID-19 global rheumatology alliance physician-reported registry. Ann Rheum Dis. 2020;79:859-66.

17. Mato AR, Roeker LE, Lamanna N, Allan JN, Leslie L, Pagel JM, et al. Outcomes of COVID-19 in patients with CLL: a multicenter international experience. Blood. 2020;136:1134-43.

Publisher's Note Springer Nature remains neutral with regard to jurisdictional claims in published maps and institutional affiliations. 